\title{
OPEN Pupal cannibalism by worker honey bees contributes to the spread of deformed wing virus
}

\author{
Francisco Posada-Florez ${ }^{1 凶}$, Zachary S. Lamas ${ }^{1,2}$, David J. Hawthorne ${ }^{2}$, Yanping Chen ${ }^{1}$, \\ Jay D. Evans $\mathbb{1}^{1 \bowtie}$ \& Eugene V. Ryabov ${ }^{1 凶}$
}

Transmission routes impact pathogen virulence and genetics, therefore comprehensive knowledge of these routes and their contribution to pathogen circulation is essential for understanding hostpathogen interactions and designing control strategies. Deformed wing virus (DWV), a principal viral pathogen of honey bees associated with increased honey bee mortality and colony losses, became highly virulent with the spread of its vector, the ectoparasitic mite Varroa destructor. Reproduction of Varroa mites occurs in capped brood cells and mite-infested pupae from these cells usually have high levels of DWV. The removal of mite-infested pupae by worker bees, Varroa Sensitive Hygiene (VSH), leads to cannibalization of pupae with high DWV loads, thereby offering an alternative route for virus transmission. We used genetically tagged DWV to investigate virus transmission to and between worker bees following pupal cannibalisation under experimental conditions. We demonstrated that cannibalization of DWV-infected pupae resulted in high levels of this virus in worker bees and that the acquired virus was then transmitted between bees via trophallaxis, allowing circulation of Varroavectored DWV variants without the mites. Despite the known benefits of hygienic behaviour, it is possible that higher levels of VSH activity may result in increased transmission of DWV via cannibalism and trophallaxis.

Pathogens, including viruses, exploit multiple transmission routes across different developmental stages which contribute to pathogen circulation and lead to diverse impacts on host physiology and life history ${ }^{1}$. Changes in modes of pathogen transmission could impose new evolutionary pressures on pathogens, in turn leading to pathogen phenotypic changes, including altered virulence ${ }^{2}$. Comprehensive models of transmission routes and their roles in pathogen circulation are essential for understanding pathogen evolutionary dynamics and development of control strategies.

Deformed wing virus (DWV) ${ }^{3}$, a principal viral pathogen of honey bees (Apis mellifera) associated with increased honey bee mortality and colony collapses ${ }^{4-6}$, has benefited from a novel transmission route in recent decades. Historically, DWV caused mainly covert infection characterized with low virus levels and transmission via food or individual bees ${ }^{7}$, but a dramatic increase of DWV virulence and infection levels was reported with the spread of ectoparasitic mite Varroa destructor, making DWV the key pathogen involved in colony collapse ${ }^{8,9}$. The mite feeds on the hemolymph and fat body tissues of pupae and adult bees ${ }^{10}$ suppressing host immunity ${ }^{11}$ and serving as effective vector for viruses, including $\mathrm{DWV}^{12,13}$. The infection of honey bees with DWV by Varroa feeding at the pupal stage can lead to the development of deformed wings ${ }^{7}$, but even asymptomatic infected bees have reduced life expectancies ${ }^{4}$. Varroa-mediated transmission of DWV by direct injection into the insect hemolymph, allowing the efficient movement of viruses from infected bees to others, has favored more virulent DWV strains. Genetic changes in DWV which occurred as a result of Varroa vectoring included reduction of genetic diversity and selection of particular strains ${ }^{14-18}$. Reproduction of Varroa mites occurs exclusively in capped honey bee brood cells, with the mite and the mite-infested pupae showing high levels of DWV ${ }^{7,12}$. Bees can suppress Varroa mite reproduction by selecting and uncapping Varroa-infested brood cells and removing infested pupae by co-called Varroa Sensitive Hygiene (VSH) ${ }^{19-21}$, a behavior enabled by the ability of bees to recognize cuticular hydrocarbon profiles characteristic for sick pupae ${ }^{22}$. It has been suggested that VSH could be accompanied by cannibalization of mite-exposed pupae ${ }^{23}$.

Cannibalism, consumption of conspecific individuals, occurs in many animals ${ }^{24,25}$. It is common in the eusocial Hymenoptera, ants ${ }^{26,27}$, wasps ${ }^{28}$, bees $^{29-31}$ and termites ${ }^{32,33}$ throughout the growth and development of

${ }^{1}$ USDA, Agricultural Research Service, Bee Research Lab, BARC-East Bldg. 306, 10300 Baltimore Ave., Beltsville, MD 20705, USA. 2Department of Entomology, University of Maryland, College Park, MD, USA. ${ }^{\square}$ email: francisco.posada@usda.gov; jay.evans@usda.gov; Eugene.Ryabov@gmail.com 
(a)

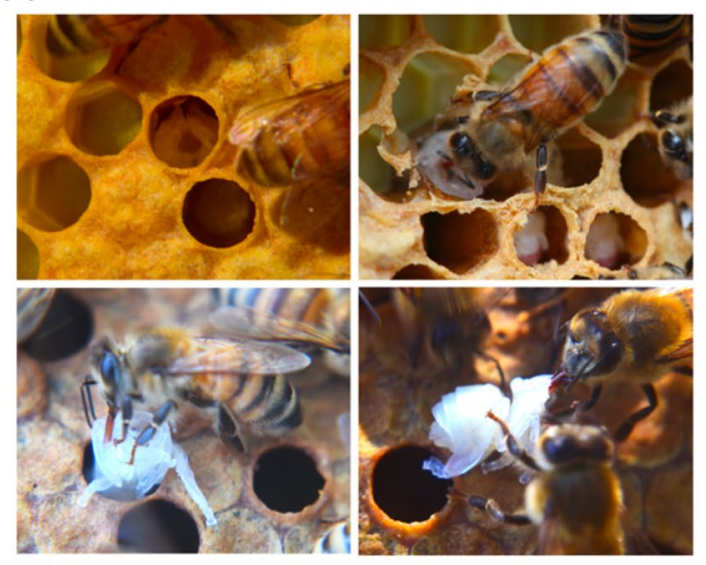

(b)

DWV in field-collected pupae

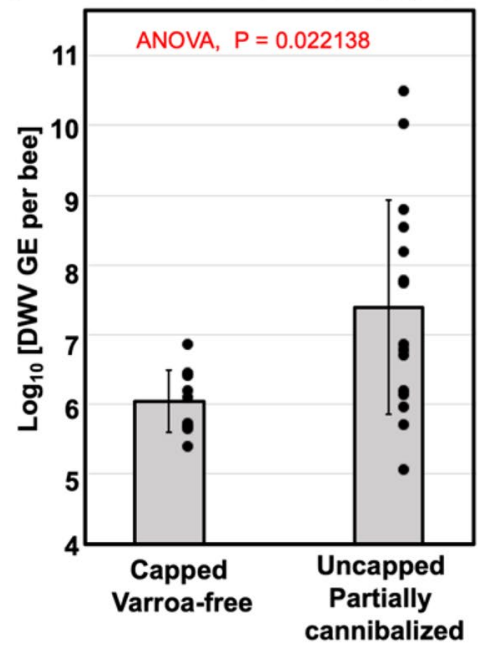

Figure 1. (a) Honey bee pupal cannibalization by worker bees. Top images: partially cannibalized pupae observed immediately after hive opening; bottom images: partially cannibalized pupae were pulled out from the cells and cannibalization continued after five minutes. (b) Average DWV RNA loads in field-collected capped and in partially cannibalized uncapped honeybee pupae, with error bars indicating standard deviation. DWV copy number in individual pupae are indicated by black dots.

the social organization and may occur for a variety of reasons including nutrient shortages, disease and pest outbreaks, environmental stressors, and colony disturbance ${ }^{34}$.

In honey bees, cannibalism is an essential part of social organization and colony-level hygiene is exercised through ecological, physiological, genetic, and sanitary stressors ${ }^{24,34-36}$. Any developmental stages and castes can be cannibalized including developing queens. Honey bees show natural cannibalism behavior when workers control worker-laid eggs ${ }^{37}$ and remove diploid drone larvae ${ }^{38}$. Cannibalization of eggs or younger larvae can be stimulated by environmental conditions, unbalanced nutrition such as scarcity of pollen ${ }^{29,31,34,39}$, and when honey bees perform hygienic behaviors ${ }^{20,21}$.

A primary risk associated with cannibalism is the increased spread of pathogens, in particular in the case of group cannibalism, i.e. when the prey is shared across a social group ${ }^{40}$. In invertebrates, ingestion of infected conspecific tissues is recognized as a route of virus transmission in insects and shrimp ${ }^{41,42}$. At the same time, reduction of the numbers of infected individuals by cannibalism might limit the spread of disease ${ }^{25}$. Although it was suggested that worker bees could be infected with DWV as a result of cannibalization of virus-infected bees ${ }^{43}$, this has not been experimentally investigated. One reason complicating the study of the impact of cannibalism on DWV circulation is the difficulty in distinguishing between DWV infection initiated by cannibalization and by other transmission routes. To solve this problem we used genetically-tagged DWV carrying unique genetic markers, the green fluorescent protein (GFP) gene and an introduced unique restriction site ${ }^{44}$, allowing us to trace transmission of the virus. We also carried out pupal cannibalism experiments in controlled laboratory conditions rather than hives, thereby minimizing virus transmission from other sources. This study provides the first direct experimental evidence that cannibalization of pupae with high levels of DWV leads to infection in worker honey bees, and that DWV could then be shared extensively among worker bees by trophallaxis. Our results suggest that cannibalization of pupae infected with DWV by Varroa mites, removed as a result of VSH activity, could provide an efficient additional route for transmission of DWV, impacting virus circulation and virulence.

\section{Results}

High levels of DWV in partially cannibalized honey bee pupae removed by hygienic activity. Partially cannibalized pupae $(n=15)$ showing different degrees of damage, ranging from partially to completely removed heads, were collected from hygienically open cells of four colonies showing cannibalism by worker honey bees (Fig. 1a). In two of these colonies, some pupae $(n=7)$ were sourced from hygienically opened brood cells containing Varroa mites at the time of collection. Notably, Varroa mites were found more often in hygienically opened brood cells containing partially cannibalized pupae than in randomly selected capped brood cells (Colony \#10: for partially cannibalized pupae 6 mite-infested and 1 mite-free, for capped cells 4 mite-infested and 84 mite-free, $\mathrm{P}<0.001$ Chi-square test for contingency table analysis; Colony \#11: for partially cannibalized pupae 1 mite-infested and 2 mite-free, for capped cells 0 mite-infested and 85 mite-free, $\mathrm{P}=0.034$ Chi-square test for contingency table analysis), suggesting that these pupal cells were opened as a result of Varroa sensitive hygienic (VSH) behaviour. We also collected control pupae $(n=9)$ from capped Varroa-free cells from areas of the brood frames where the partially cannibalized pupae were sourced. The pupae of both the control and damaged groups were at white to pink-eye developmental stages. Quantification of DWV RNA by RT-qPCR (Fig. 1b, Supplementary Table 1) showed that the levels of DWV in the partially cannibalized pupae (range 5.05-10.50 $\log _{10} \mathrm{GE} /$ pupa; $7.39 \pm 1.59 \log _{10}$ genome equivalents (GE)/pupa, mean $\pm \mathrm{SD}$ ) were significantly 
(a)

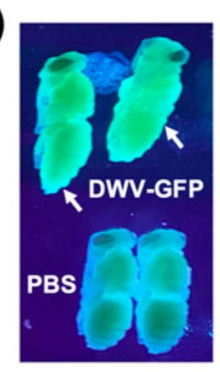

(b) Experiment A: Cannibalism of Varroa-infected pupa

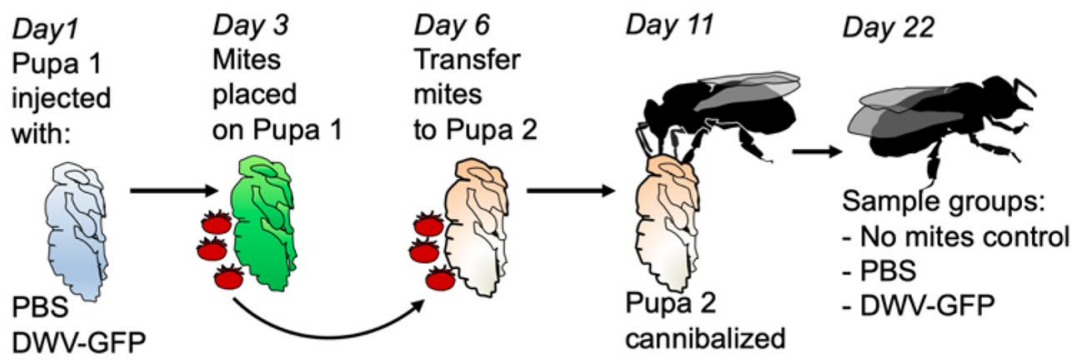

(c)

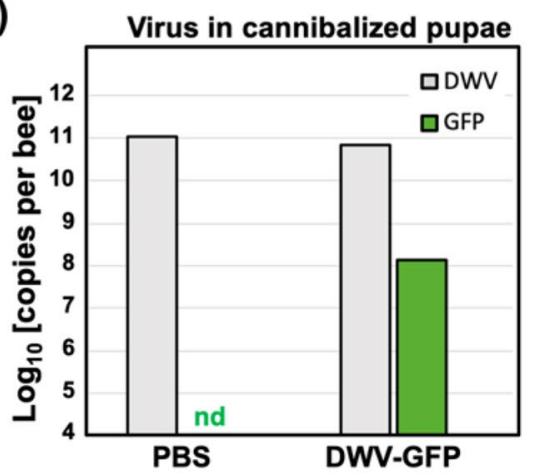

(d) Worker bees, 11 days post cannibalization

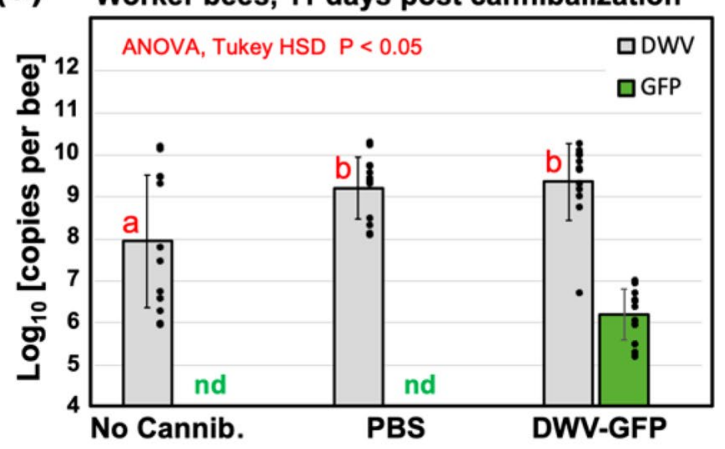

(e)

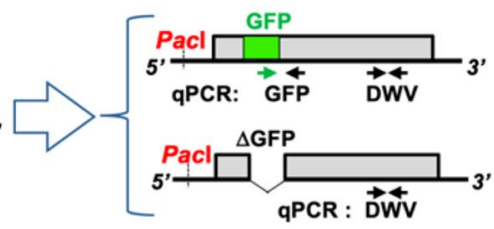

Figure 2. DWV infection in worker bees following cannibalization of pupae infected by Varroa mites (Experiment A). (a) Honey bee pupae, control and DWV-GFP-infected (pointed with arrows) which were used to rear Varroa mites (Pupae 1), illuminated with $395 \mathrm{~nm}$ UV light; (b) Schematic representation of the experiment. (c) DWV and GFP RNA loads in Varroa exposed Pupae 2 offered for cannibalization. (d) Average DWV and GFP RNA loads in individual worker bees, error bars indicate standard deviation. For DWV, red letters above bars indicate significantly and non-significantly different groups (ANOVA). DWV and GFP copy number in individual pupae are indicated by black dots, $n d$ not detectable levels. (e) Schematic representation of the DWV-GFP RNA genome and genetic changes following deletion of the GFP-coding sequence, positions of qPCR primers used for quantification of DWV and GFP RNA and genetic changes in DWV-GFP following deletion of the GFP-coding sequence are indicated.

higher than in the capped Varroa-free pupae (5.39-6.86 $\log _{10}$ GE/pupa; $6.05 \pm 0.47 \log _{10}$ GE/pupa, mean $\pm S D$ ), $\mathrm{P}=0.022, \mathrm{df}=23$, ANOVA (Fig. 1b). There was no significant difference between these groups in the levels of honey bee actin mRNA $(\mathrm{P}=0.560, \mathrm{df}=23$, ANOVA) (Supplementary Table 1$)$ confirming that no tissue degradation, potentially affecting RNA quality or actin expression, took place in the damaged pupae.

Acquisition of DWV by worker bees as a result of cannibalism of pupae infected by Varroa. Experiment A tested if cannibalization of the pupae infected with DWV by Varroa mites could result in development of the virus infection in worker bees. It included injecting honey bee pupae with a filtered tissue extract containing DWV-GFP particles ${ }^{44}$, or by a phosphate buffer saline (PBS) control. After $48 \mathrm{~h}$, when GFP fluorescence had developed in the DWV-GFP-injected pupae indicating virus infection (Fig. 2a), Varroa mites were placed on the pupae and reared for $72 \mathrm{~h}$ to acquire the virus ${ }^{45}$. Then, these mites were transferred to new white-eye pupae (Fig. 2b, Pupa 2) which were reared for 5 days to allow transmission of the virus from Varroa and development of infections in the recipient pupae. Pupae from both PBS and DWV-GFP groups were cut into two pieces along the longitudinal plane, one half was used to extract RNA for molecular analysis of the DWV and GFP loads (Fig. 2c), and another half was offered to a group of 20 worker bees 4 days post-emergence (Fig. 2b). A control group of 20 worker bees received no pupae for cannibalization. Nearly complete cannibalization of "PBS" and "DWV-GFP" pupal tissues was observed after $12 \mathrm{~h}$ incubation. The worker bees were maintained for an additional 10 days before sampling for molecular analysis of virus loads (Fig. 2b).

RT-qPCR analysis of pupae which were exposed to Varroa mites for 5 days revealed that both "PBS" and "DWV-GFP" pupae had high levels of DWV RNA (Fig. 2c) (11.03 $\log _{10}$ GE/pupa, and $10.83 \log _{10}$ GE/pupa, correspondingly). GFP RNA was detected only in pupae ( $\left.8.13 \log _{10} \mathrm{GE} / \mathrm{pupa}\right)$ which were exposed to the mites 
(a) Experiment B. Trophallactic transmission

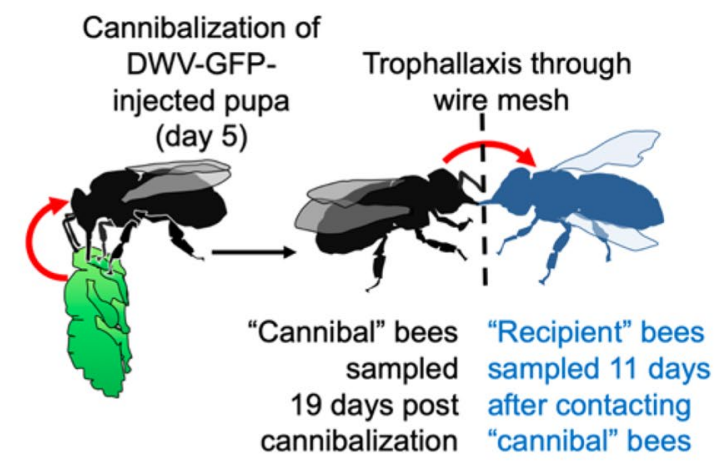

(b)

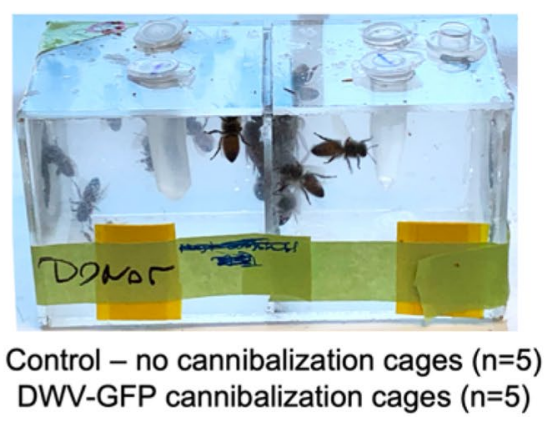

(c)

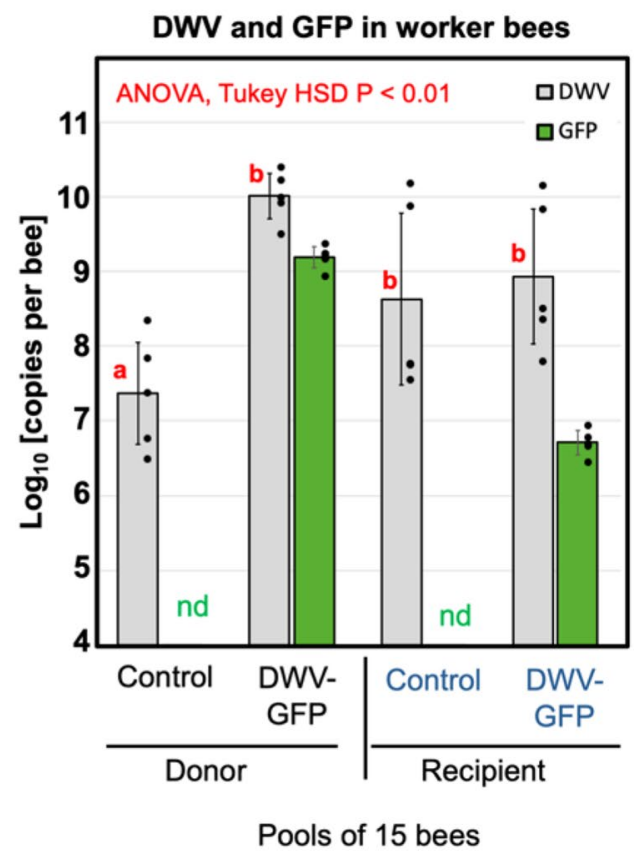

Figure 3. Trophallactic transmission of cannibalized DWV (Experiment B). (a) Schematic representation of the experiment. (b) Design of the trophallactic cages. (c) Average per insect levels of DWV and GFP RNA in the pools of worker bees, error bars indicate standard deviation. For DWV, red letters above bars indicate significantly and non-significantly different groups (ANOVA). Average DWV and GFP copy number in the cage pools are indicated by black dots, nd not detectable levels.

which acquired DWV-GFP (Fig. 2d). The observed 496-fold (2.695 $\left.\log _{10}\right)$ excess of DWV over GFP in Pupa \#2, which received DWV-GFP by mite transmission, could be a result of both accumulation of clone-derived DWV genomes with deletion of the GFP-coding sequence ${ }^{44}$ and transmission of wild-type DWV by Varroa mites. Indeed, high DWV levels in Pupa 2 of "PBS treatment" (Fig. 2c) suggested transmission of wild-type DWV by the Varroa mites used in this experiment.

Analysis of virus levels in worker bees 11 days post cannibalization (dpc) showed that "PBS" and "DWV-GFP" groups had similar levels of DWV $\left(9.21 \pm 0.74 \log _{10}\right.$ GE/bee, and $9.35 \pm 0.91 \log _{10}$ GE/bee, mean \pm SD, correspondingly). DWV load in worker bees of the control group, which did not cannibalised pupa, "No Cannib.", $7.94 \pm 1.58$ $\log _{10} \mathrm{GE} /$ bee, mean $\pm \mathrm{SD}$, were significantly lower than in the groups which consumed DWV infected pupal tissue $(\mathrm{P}<0.05, \mathrm{df}=35$, ANOVA) (Fig. 2d, Supplementary Table 1). GFP RNA was present in the "DWV-GFP" worker bees at the levels of $6.20 \pm 0.61 \log _{10} \mathrm{GE} / \mathrm{bee}$, mean $\pm \mathrm{SD}$, which was lower than in the cannibalised "DWV-GFP" Pupa 2. The observation of an average 2502-fold (CI 951030 to 6081-fold) excess of DWV over GFP in worker bees in the "DWV-GFP" treatment group could be explained by further loss of the GFP-coding sequence from the clone-derived DWV-GFP (Fig. 2e) and also by the presence of wild-type DWV.

Trophallactic transmission of the virus acquired by pupal cannibalism. Experiment A (Fig. 2) demonstrated that pupae infected with DWV by Varroa mites could act as a source of infection when cannibalized by worker bees. The levels of DWV in these pupae, $10 \log _{10}$ to $11 \log _{10}$ GE (Fig. 2c), were similar to those observed in the pupae infected with DWV-GFP by injection ${ }^{44}$. Therefore, such injection-infected pupae could be used as an adequate surrogate for Varroa-infected pupae in cannibalism experiments.

The impact of pathogens acquired by cannibalization depends on the number of individuals involved in cannibalism, either directly or through sharing the infected tissues ${ }^{40}$. Worker honey bees always exchange food by trophallaxis, which could allow the virus from the cannibalized tissues to spread to a large number of workers. To test if such transmission takes place we devised an Experiment B (Fig. 3a) to investigate transmission of the infection between groups of worker bees separated by a wire mesh, allowing trophallactic contacts but not bee movement (Fig. 3b). A white-eyed pupa injected with DWV-GFP inoculum (7 $\log _{10}$ GE), which showed GFP fluorescence consistent with $10 \log _{10}$ to $11 \log _{10} \mathrm{GE}$ of the virus $48 \mathrm{~h}$ after injection (hpi), was divided into 5 equal parts, which were offered to 5 groups of 25 worker bees in the donor (cannibal) chambers of the cages. Controls, 5 groups of 25 worker bees, did not receive pupal tissue. Both control and experimental worker bees were 4 days old and were sourced from colony \#2 with $0.5 \%$ Varroa mite infestation rate. Complete or nearly complete cannibalization of the offered pupal tissues was observed in each of 5 experimental cages. Five days later, newly emerged worker bees were placed into the "Recipient" chambers of all 10 cages (Fig. 3b) and were 
maintained for an additional 10 days before sampling. The donor and recipient cages contained sugar syrup feeders, but to promote trophallaxis from the donor cage workers, the feeders were removed from the recipient cages for $8 \mathrm{~h}$ during the first 3 days after introduction of the bees.

Average per-bee loads of DWV and GFP RNA were quantified by RT-qPCR in pools of 15 worker bees which were sampled from each donor chamber 19 days post cannibalism (dpc), and from each recipient chamber 11 days after contacting donor bees (Fig. 3c). Overall DWV levels, which included wild-type DWV, DWV-GFP and GFP deletion variants of this virus, were not significantly different in both recipient groups ("Control" and "DWV-GFP") and in the donor "DWV-GFP" group (8.63 $\pm 1.15 \log _{10}$ GE/bee, $8.93 \pm 0.90 \log _{10}$ GE/bee, and $10.01 \pm 0.30 \log _{10} \mathrm{GE} / \mathrm{bee}$, mean $\pm \mathrm{SD}$, respectively) while DWV levels were significantly lower $(\mathrm{P}<0.01, \mathrm{ANOVA})$ in the donor "control" group, $7.37 \pm 0.68 \log _{10}$ GE/bee (Fig. 3c, Supplementary Table 1). The presence of GFP in the bees of both "Donor-DWV-GFP" and "Recipient-DWV-GFP" groups $\left(9.19 \pm 0.14 \log _{10}\right.$ GE/bee and $6.71 \pm 0.16 \log _{10} \mathrm{GE} /$ bee, mean $\pm \mathrm{SD}$, respectively) but not in the control bees confirmed development of DWVGFP infection following cannibalization of infected pupal tissues and further transmission of the tagged virus via trophallaxis (Fig. 3c, Supplementary Table 1). High levels of DWV in both control recipient groups was likely a result of a wild-type DWV infection, which was present in the recipient bees. Such contamination with wild type virus was not surprising because DWV is widespread in Maryland colonies ${ }^{18}$ and it is known that newly emerged worker honey bees may develop DWV infection even without receiving additional virus inoculum ${ }^{46}$. Although the levels of GFP were approximately 7 times lower than those of DWV in the donor "DWV-GFP" groups (Fig. 3c), the PacI restriction analysis of the RT-PCR fragment for the $5^{\prime}$ terminal region showed that all DWV present in these bees derived from DWV-GFP (Fig. 4a, lanes "Ex-B-Donor"). In the recipient "DWVGFP" group, DWV levels were 167 -fold higher than those of GFP, indicating that no more than $0.6 \%$ of the population contained intact DWV-GFP (Fig. 3c). At the same time, the PacI digestion test which targeted the clone-derived DWV (Fig. 2e) showed that $42 \%$ of the virus in the recipient "DWV-GFP" group derived from DWV-GFP (Fig. 4a, lanes "Exp-B-Recipient”).

DWV to GFP ratios, an indicator of the GFP loss from DWV-GFP, were increased in the recipient group compared to the donor group (Figs. 3c, 4b). This change was estimated as 25 -fold for overall DWV, or tenfold when only DWV-GFP-derived virus containing PacI was considered and was statistically significant $(\mathrm{P}=0.02196$ for overall DWV levels, $\mathrm{P}=0.07236$ for the DWV-GFP-derived alone).

Dynamics of DWV infection in worker bees following pupal cannibalism. Experiment $C$ further investigated replication of DWV in worker bees at the individual insect level following consumption of DWVinfected pupae and further transmission of infection between worker bees when full contact was possible, similar to natural interactions between worker bees in the hive (Fig. 5a). This experiment involved three groups of 200 four-day old worker bees. The treatments included: no cannibalization control (Groups C), cannibalization of a Varroa-free pink eye pupae from the colony \#2 with low levels of wild-type DWV, 95\% Confidence Interval (CI) 5.35-5.75 $\log _{10}$ GE/pupa (Group T1), and cannibalization of the DWV-GFP injected pupa with high levels of the virus (95\% CI 10.70-11.03 $\log _{10}$ GE/pupa; Group T2). DWV and levels of GFP were determined by RTqPCR in individual bees 4,8 and $12 \mathrm{dpc}, 10$ insects were collected from each group for every sampling event. To investigate trophallactic transmission, 10 groups of 10 bees from each treatment group were collected at $4 \mathrm{dpc}$, marked and placed into the cages containing 90 newly emerged bees and reared together for an additional 8 days. Then, the groups of 50 unmarked bees were collected and pooled for each of 30 cages and the levels of DWV and GFP were quantified. Molecular analysis showed that cannibalization of a Varroa-free honey bee pupa by the T1 bees did not result in development of a high-level virus infection in worker bees, and remained the same as in the control group which did not cannibalize (C) and as in the bees at the start of the experiment (T0), Fig. 3b. No GFP RNA was detected in the bees of T0, C and T1 of the "Cannibal" groups at any timepoint (Fig. 5b) and in C and T1 "Recipient" pools (Fig. 5c) (Fig. 5b). Cannibalisation of DWV-GFP infected pupae resulted in the development of DWV-GFP infection in worker bees (Fig. 5b, groups T2). At 4 dpc, the levels of DWV in T3 group were significantly higher than in the control group $\mathrm{C}$ and in the T1 groups which cannibalised non-injected pupa (Fig. 5b), ranging from 6.37 to $8.17 \log _{10} \mathrm{GE} / \mathrm{bee}\left(7.63 \pm 0.46 \log _{10} \mathrm{GE} / \mathrm{bee}\right.$, mean $\left.\pm \mathrm{SD}\right)$. Similarly, high levels of GFP RNA were observed in 9 out of 10 bees, reaching $8.15 \log _{10}$ GE/bee $\left(7.37 \pm 1.14 \log _{10}\right.$ GE/ bee, mean $\pm \mathrm{SD}$ ), with a single bee in this group having undetectable levels of GFP and DWV loads similar to those in bees of groups C and T1, $6.368 \log _{10}$ GE/bee. Such nearly uniform distribution of DWV-GFP among 200 bees in the T2 group suggests that a high proportion of bees was involved in cannibalism and/or sharing of the virus-infected pupal tissue by trophallaxis. DWV-GFP infection continued to develop in T2, bees, exceeding $10^{9}$ copies per worker at $8 \mathrm{dpc}$ ( 4 out of 10 sampled bees, highest level $10.62 \log _{10} \mathrm{GE} / \mathrm{bee}, 8.80 \pm 1.11 \log _{10} \mathrm{GE} /$ bee, mean $\pm \mathrm{SD}$ ), and maintaining these levels at $12 \mathrm{dpc}$ (with 2 out of 10 sampled bees, highest level 10.63 $\log _{10} \mathrm{GE} /$ bee $\left(8.36 \pm 1.08 \log _{10} \mathrm{GE} /\right.$ bee, mean $\pm \mathrm{SD}$ ) (Fig. $5 \mathrm{c}$ ). The GFP RNA loads in "Cannibal T2" groups at 8 and $12 \mathrm{dpc}\left(8.51 \pm 0.73 \log _{10} \mathrm{GE} /\right.$ bee and $8.14 \pm 0.79 \log _{10} \mathrm{GE} /$ bee, mean $\left.\pm \mathrm{SD}\right)$ in these bees were slightly lower than those of DWV RNA (Fig. 5b). However, it was demonstrated by the complete digestion of cDNA fragments corresponding to the $5^{\prime}$ regions of DWV RNA with PacI demonstrated that DWV, which did not carry the GFP insert, derived from DWV-GFP (Fig. 4a, lines "Ex-C-Cannibal-4, -8, -12 dpc").

Experiment $\mathrm{C}$ also tested the ability of bees which acquired DWV-GFP by cannibalization to transmit the virus to naïve worker bees when they are reared together. This was done by collecting 10 groups of 10 marked bees from each of three Donor cages at 4 days after cannibalisation. The worker bees were marked and then transferred to Recipient cages containing 90 naïve newly emerged worker bees after which recipient bees were reared for 8 days (Fig. 5a). Molecular analysis of DWV and GFP RNA loads was carried out for the pool of 50 recipient unmarked bees for each of 30 Recipient cages (10 for each of 3 groups). The highest levels of DWV $\left(9.43 \pm 1.01 \log _{10} \mathrm{GE} / \mathrm{bee}\right.$, mean $\pm \mathrm{SD}$, range $\left.6.75-10.84 \log _{10} \mathrm{GE} / \mathrm{bee}\right)$ were observed in the cages of the T2 group 
(a)

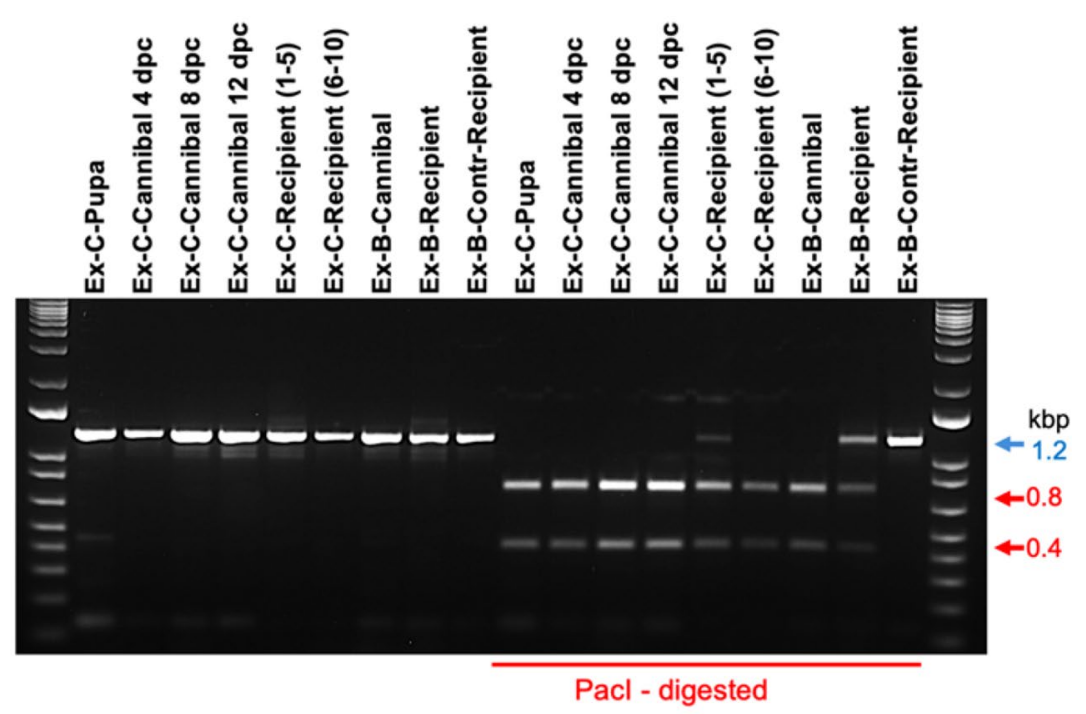

(b)

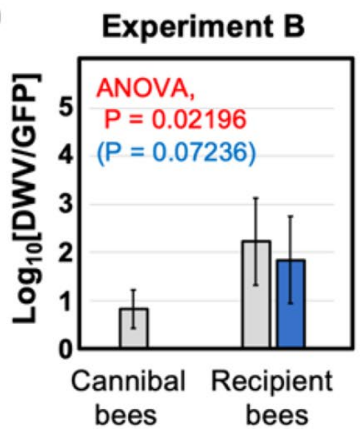

(c)

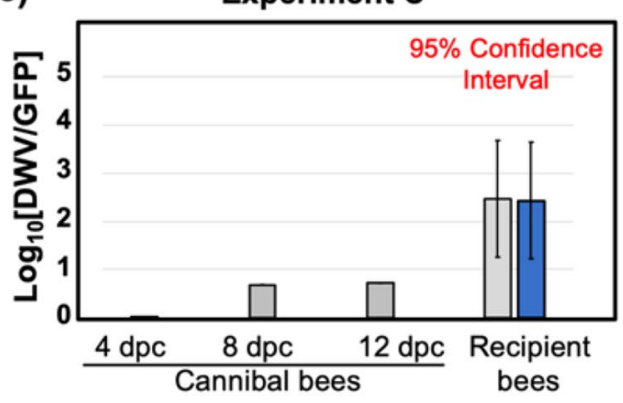

Figure 4. Dynamics of DWV-GFP in worker bees following cannibalism and trophallactic transmission. (a) Identification of DWV-GFP-derived viral progeny in the treatment group pools of Experiments B and C. The analysis included amplification of a 1237 nt RT-PCR fragments corresponding to the 5 '-terminal region of DWV genome, digestion with $\mathrm{PacI}$, and separation of the digestion reaction products by agarose gel electrophoresis. The untreated $1237 \mathrm{nt}$ fragments (left) and PacI-digested (right). The digestion fragments (left) derived from DWV-GFP, expected fragment sizes, undigested (blue arrow) and digested (red arrows), are shown on the right. Treatment groups are shown above, prefixes "Ex-B-" and "Ex-C-" indicate samples of the Experiment $\mathrm{B}$ and $\mathrm{C}$ treatment groups, respectively. Two pools of 5 Recipient cages were analyzed for Experiment C. (b,c) Accumulation of GFP deletion variants derived from the DWV-GFP genome in the recipient bees which received the virus by trophallaxis from "Cannibal" bees. Columns indicate ratios between DWV RNA load and GFP RNA load in a sample, grey columns-for overall DWV levels, blue columns-for DWV originated from DWV-GFP (when wild-type DWV without PacI site was present). Error bars indicate (b) standard deviation or (c) 95\% Confidence Interval, for (b) ANOVA P-values for uncorrected DWV load (red) and corrected DWV load (blue) are shown.

which received bees that cannibalized DWV-GFP infected pupa. Levels of DWV in T2 Recipient cages were significantly higher $(\mathrm{P}<0.01$, ANOVA, Tukey's HSD) than those of the Control C group which did not cannibalise pupae (average $5.24 \pm 0.69 \log _{10} \mathrm{GE} /$ bee, mean $\pm \mathrm{SD}$, range $4.15-6.39 \log _{10} \mathrm{GE} / \mathrm{bee}$ ) or T1 group, which cannibalised Varroa-free pupa (average $5.31 \pm 0.87 \log _{10} \mathrm{GE} / \mathrm{bee}$, mean $\pm \mathrm{SD}$, range 4.71-7. $29 \log _{10} \mathrm{GE} / \mathrm{bee}$ ) (Fig. 5c). GFP targets were detected exclusively in T2 group cages, $6.93 \pm 0.87 \log _{10} \mathrm{GE} / \mathrm{bee}$, mean $\pm \mathrm{SD}$, range 4.98-7.64 $\log _{10}$ GE/bee (Fig. 5c), indicating transmission of DWV-GFP acquired by cannibalism. Analysis of the RT-PCR fragment corresponding to the $5^{\prime}$ region of DWV populations from group T2 showed that $93 \%$ of DWV contained the PacI restriction site unique for the cDNA clone-derived virus, indicating that a majority of the virus population originated from DWV-GFP. At the same time, RT-qPCR showed that DWV to GFP ratios in Recipient T2 groups were approximately 290:1 $\left(2.46 \log _{10}\right)$, (or 268:1 $\left(2.43 \log _{10}\right)$ if only clone-derived virus was considered. The DWV to GFP ratios in the T2 Cannibal bees were well below the $95 \%$ confidence limit for DWV to GFP ratios for T2 Recipient cages (Fig. 4c). In the Cannibal T2 group, nearly equimolar levels of DWV and GFP were observed at $4 \mathrm{dpc}$. As infection developed, accumulation of the viral variants with the deletion of GFP-coding sequence resulted in increase of the DWV to GFP ratios at $8 \mathrm{dpc}$ and $12 \mathrm{dpc}$ to 4.5 and 5.2, respectively (to $0.69 \log _{10}$ and $0.72 \log _{10}$, respectively) (Fig. 4c). 
(a) Experiment C: Infection dynamics and transmission

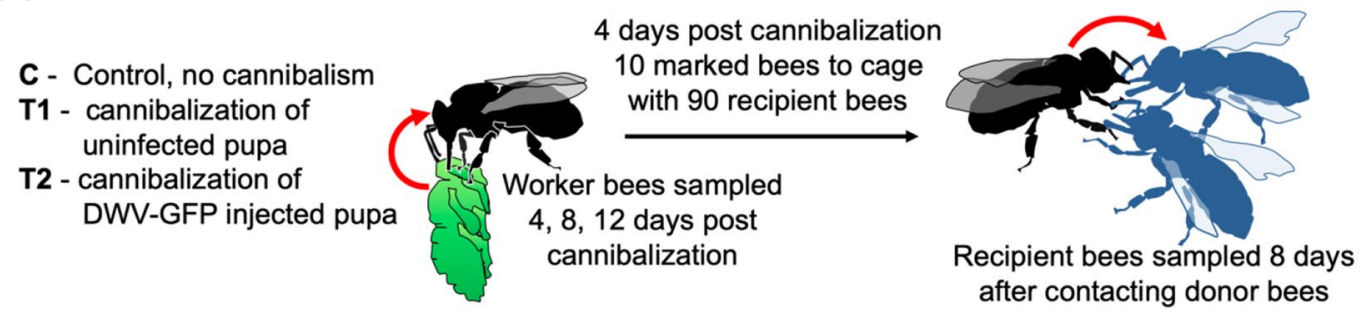

(b)

Donor cages

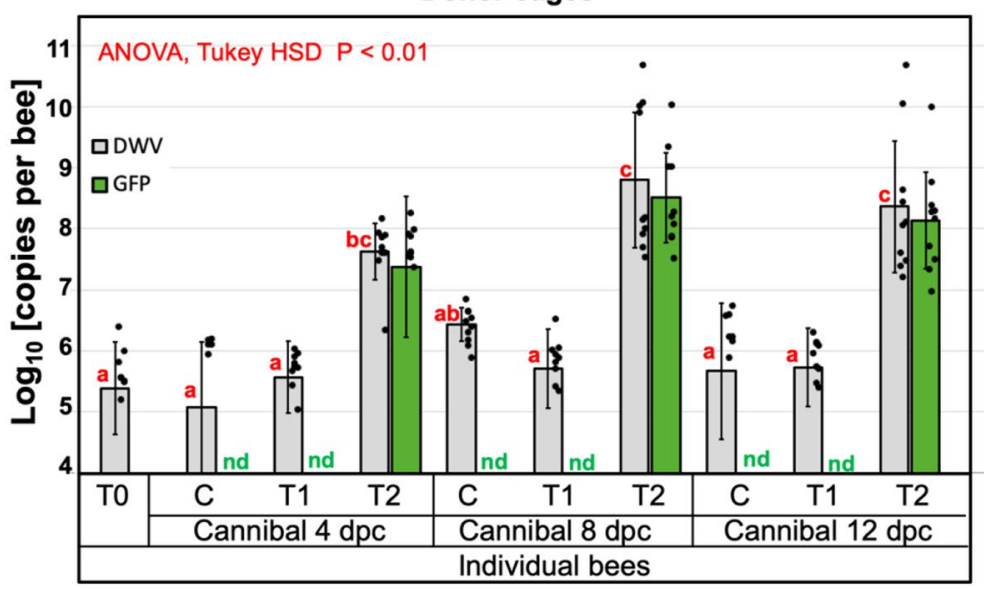

(c)

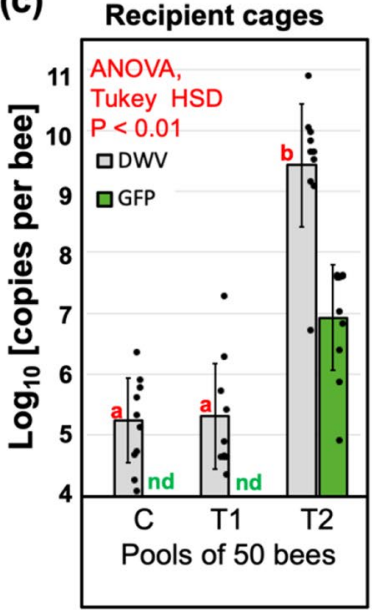

Figure 5. DWV dynamics in worker bees following cannibalization (Experiment C). (a) Schematic representation of the experiment. (b) Average DWV and GFP RNA loads in individual worker bees of the "cannibal" group, error bars indicate standard deviation. For DWV, red letters above bars indicate significantly and non-significantly different groups (ANOVA). (c) Average DWV and GFP RNA loads in the pools of worker bees of the recipient groups, error bars indicate standard deviation. For DWV, red letters above bars indicate significantly and non-significantly different groups (ANOVA). DWV and GFP copy number in individual pupae are indicated by black dots, $n d$ not detectable levels.

\section{Discussion}

High-throughput sequencing has allowed the comprehensive characterization of invertebrate viromes, allowing the discovery of many novel viruses ${ }^{47}$, but understanding virus biology, including transmission routes, is lagging behind. This study investigated impacts of cannibalization of pupae by adult worker bees on circulation of DWV, the principal viral pathogen of honey bees ${ }^{3}$. Pupae cannibalized in Varroa-infested colonies were likely to be uncapped as a result of Varroa sensitive hygienic activity (Fig. 1), and some of these partially cannibalized pupae were shown to contain high levels of DWV consistent with overt DWV infections (Fig. 1b). Importantly, in approximately half of the hygienically removed pupae the DWV levels were similar to those in the uncapped Varroa-free control. This could be a result of removal of infected pupae at early stages of infection, and it is known that hygienic bees have a lower threshold for detection of the infection scent ${ }^{48}$.

Testing the spread of DWV in colonies has proved to be difficult because it was not previously possible to distinguish between virus acquired by worker bees via cannibalism or via other routes, considering the nearly ubiquitous spread of DWV. Therefore, the role of cannibalism in maintenance of DWV infection has remained speculative so far. To test this hypothesis, we investigated cannibalism and transmission in controlled experimental conditions, using genetically tagged DWV isolate that allowed us to trace infections. This tagged virus containing a GFP insert ${ }^{44}$ was based on the cDNA clone of a virulent DWV isolate originated from Varroainfested pupae sourced from a dying colony ${ }^{18}$, therefore this variant is suitable to study transmission of DWV acquired as a result of hygienic removal and cannibalization of Varroa-infested pupae.

We demonstrated that cannibalization of honey bee pupae infected with DWV either by Varroa mites (Fig. 2) or artificially infected with this virus by injection (Figs. 3 and 5), which contained high levels of the virus (95\% CI 10.70-11.03 $\log _{10}$ GE per pupa), resulted in infection levels typical for overt DWV infection, above $9 \log _{10}$ GE per insect ${ }^{18}$, reaching $10.84 \log _{10} \mathrm{GE}$, in worker bees at 8 dpc (Figs. 3 and 5). The levels of DWV-GFP in the pupae, cannibalization of which resulted in development of infections in workers, (Fig. 2c) were similar to those in some partially cannibalized pupae which were uncapped in hives as a result of VSH activity (Fig. 1) indicating that infection of workers as a result of pupal consumption could take place under natural hive conditions. Therefore virus-infected cannibalized pupae could act as "superspreaders" infecting large number of worker bees. For example, Experiment $C$ showed that after cannibalization of a single pupa in a cage with 200 worker bees, 24-148 bees (CI 95 12.2-73.8\%) had developed overt DWV levels (Fig. 5b). At the same time, Experiment 


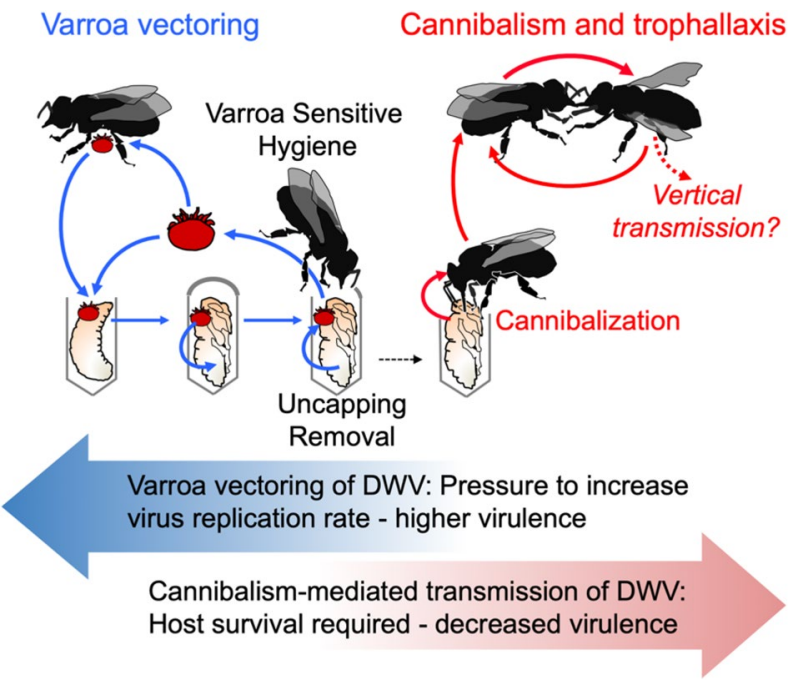

Figure 6. Model of DWV circulation in Varroa mite-infested Varroa Sensitive Hygienic (VSH) colonies. Varroa transmission-blue arrows, cannibalism-trophallaxis transmission-red arrows. Block arrows show possible evolutionary pressures which on Varroa and cannibalism-trophallaxis transmission routes impose on DWV virulence.

C showed that cannibalization of pupae with low levels of DWV, typical for covert infections, with $95 \% \mathrm{CI}$ 5.35-5.75 $\log _{10}$ GE/pupa, did not result in development of overt level infection in worker bees (Fig. 5c, Group T1). It is possible that honey bees are adapted to suppress development of infection when tissues with low DWV are acquired orally ${ }^{17}$, but are unable to resist infection when high doses are ingested via cannibalized pupal tissue. Interestingly, hygienic bees are known to be more sensitive to cues from infected bees ${ }^{48}$, which might allow the removal and cannibalization of pupae with lower pathogen levels and, hence, lower risk for hygienic workers. Pupae with high DWV levels (above $9 \log _{10}$ GE) are associated with Varroa mite infestation. Considering that Varroa became a parasite of A. mellifera very recently, it is possible that $A$. mellifera has not evolved antiviral defenses that might allow them to withstand high viral doses orally.

Considering that nurse worker honey bees are actively exchanging consumed food from mouth to mouth by trophallactic interactions ${ }^{49}$, such transmission of DWV acquired by cannibalism was tested in Experiments B and C (Figs. 3, 5). It was demonstrated that the virus was readily transmitted by trophallaxis from cannibalistic bees to naïve recipient worker bees, 8 days after the cannibalistic bees and naïve bees were in full contact (Experiment C, Fig. 5) or being separated by a wire mesh screen allowing trophallactic contact only (Experiment B, Fig. 3). Development of overt virus infection in a large number of recipient worker bees (Fig. 3c, "DWV-GFP"; Fig. 5c T2 group) was observed. Efficient spread of DWV-GFP via trophallaxis, when a single infected pupa was effectively shared between 2000 worker bees (Fig. 5), was in a good agreement with the spread of radioactively labelled sugar syrup, which showed that food taken by a single bee could be shared to hundreds and thousands of foragers and nurses within 1 day $^{50}$. Taken together, these findings suggest that cannibalism combined with trophallaxis allows effective spread of DWV between worker bees (Fig. 6). This is an important finding which showed that even if a small proportion of the workers were actively engaged in cannibalism, the infected tissue could be shared between large numbers of the workers in the colony ${ }^{50}$. Such sharing could increase the impacts of cannibalism on DWV circulation ${ }^{40}$. Further studies of this phenomenon should be carried out in natural colonies, in particular with bees showing different levels of hygienic behaviour ${ }^{51}$, to better determine the risk to colonies via this cannibalism-trophallactic route.

The use of GFP-tagged DWV gave an additional insight into the mechanisms of trophallactic transmission of DWV. The clone-derived DWV included a non-essential GFP gene, which could be lost from viral genomes during replication ${ }^{44}$ (Fig. 2e). Following replication of this DWV-GFP clone-derived virus population, the proportion of viral genomes with the GFP deletion increased and the loss of GFP could be utilized as a molecular clock. This allowed us to distinguish between the original virus (with nearly 1:1 ratio of DWV to GFP copies) and virus populations which had gone through multiple cycles of replication ${ }^{44}$ (Fig. 2e). Therefore, the higher DWV to GFP copy number ratio in worker bees which acquired the virus by trophallaxis from the bees involved in cannibalism (Fig. 4b) suggested transmission of the virus produced after replication events in the worker bees rather than directly from the cannibalized pupal tissues. It is known that hypopharyngeal and mandibular gland secretions of the worker bees could be shared by trophallaxis ${ }^{30,52}$ and DWV was detected in hypopharyngeal glands of worker bees ${ }^{53}$. Notably, the transmission experiments (Figs. 3 and 5) were designed to include cannibal (donor) bees at the peak of the hypopharyngeal gland activity, 8 days post emergence ${ }^{54}$. Efficient transmission and circulation of cannibalism-acquired DWV therefore, could depend on the survival of the infected bees, thereby selecting against DWV virulence (Fig. 6). Natural attenuation has been reported for RNA viruses, including flaviviruses Japanese encephalitis virus and Dengue virus type- $2^{55,56}$. 
Our results suggest that cannibalization of Varroa-infested pupae uncapped as a result of VSH activity and trophallactic interactions could provide an efficient route for transmission and circulation of a Varroa-vectored DWV (Fig. 6). While it was shown that higher VSH activity resulted in lower levels of DWV in the colonies ${ }^{57,58}$, the cannibalism-trophallactic transmission route shows that the virus could start circulating in the colony following successful VSH removal and cannibalization of the Varroa-infested pupae and is not immediately eliminated. It is possible that colonies could tolerate a certain level of such additional virus input. This suggestion is supported by observation that the amount of time during which colonies experienced high Varroa levels was positively correlated with colony mortality ${ }^{59}$. Also, the cannibalism-trophallactic transmission route would allow quick spread of new DWV variants introduced as a result of introduction of Varroa mites on bees from different colonies during drifting and robbing ${ }^{60}$. Oral acquisition of infected pupal tissues with high virus loads and further trophallactic transmission results in DWV infection with the levels typical for overt infection of the virus in the worker bees. This might explain why high DWV loads persist and a poor survival prognosis remains in the colonies which reached a threshold Varroa infestation level, even if Varroa mites are eliminated via varroacide treatments ${ }^{12}$. While VSH is an important trait for reducing mite parasitism, this study suggests that increased VSH activity in Varroa-infested hives could lead to increased infection levels and circulation of DWV. Therefore, the cannibalism-trophallactic transmission route of DWV, in addition to Varroa vectoring (Fig. 6), should be considered in designing anti-Varroa and antivirus treatments of honey bees.

\section{Methods}

Analysis of field-collected partially cannibalized pupae. Partially cannibalized and capped worker honey bee pupae were sourced from honey bee colonies maintained in Patuxent Research Refuge $\left(39^{\circ} 00^{\prime} 56.6^{\prime \prime} \mathrm{N}\right.$ $\left.76^{\circ} 49^{\prime} 05.3^{\prime \prime} \mathrm{W}\right)$, Beltsville, Maryland, in July 2020. The colonies were not treated against Varroa mites during the year 2020 and had $3-5 \%$ of Varroa mite infestation per worker bee. Collected pupae were frozen at $-80^{\circ} \mathrm{C}$ within $30 \mathrm{~min}$ after collection and were maintained frozen at this temperature prior to RNA extraction.

Honey bees and Varroa mites. The worker bees used in laboratory cannibalism experiments were sourced in June 2020 from the Beltsville USDA Beltsville Bee Research Laboratory apiary $\left(39^{\circ} 02^{\prime} 31.8^{\prime \prime} \mathrm{N} 76^{\circ} 51^{\prime} 52.6^{\prime \prime} \mathrm{W}\right)$ from a strong colony JC-2 for Experiments A, C, and B (cannibal group), and JC-6 for Experiment B (recipient bees). These colonies had low Varroa mite infestation rates (below $0.5 \%$ ), and the DWV loads in their pupae were undetectable by qRT-PCR in May and June 2020. To obtain newly emerged workers, the frames from these colonies with sealed brood close to emergence were placed in cages in an environmental chamber set to $32{ }^{\circ} \mathrm{C}$ and $85 \%$ relative humidity in darkness, and newly emerged adult bees were collected after $18 \mathrm{~h}$ of incubation. This allowed for reduced exposure of newly emerged bees to DWV in the source colony. Pupae at the white-eyed stage were pulled out of Varroa-free cells of colony JC-2 using soft tweezers no more than $24 \mathrm{~h}$ prior to their use in the experiments. Varroa mites were manually collected from newly emerged drones sourced from additional Varroa-infested colonies in the BRL apiary. Varroa mites were hand-collected from adult drones from the broodnest of colonies maintained in College Park, MD and the USDA. The colonies had high varroa levels but did not show clinical signs of varroosis. Cannibalism experiments were carried out in dark incubators, at $+33^{\circ} \mathrm{C}$, relative humidity $85 \%$ relative humidity. Transmission experiments were carried out in custom trophallaxis cages (Fig. 3b) designed and fabricated by ZSL. The worker bees had ad libitum access to sugar syrup in a 1:1 ratio accessible and water in the tube feeders changed every $24 \mathrm{~h}$. For RNA extraction, live bees were sampled and immediately frozen at $-80^{\circ} \mathrm{C}$. In each experiment there were no significant differences in worker bee mortality between treatment groups.

Infection of honeybee pupae by DWV-GFP. Honey bee pupae at the white eye developmental stage collected from Varroa-free brood cells were injected with $8 \mu \mathrm{L}$ of a filtered extract containing $7 \log 10$ of DWV-GFP virus particles. This extract was generated using individual pupae infected with in vitro RNA transcript from the construct pDWV-L-GFP carrying an enhanced-GFP coding sequence ${ }^{44}$, which gave an equimolar ratio of DWV to GFP in qRT-PCR tests, indicating that it contained mainly intact recombinant virus without GFP deletions. The extract-injected pupae were incubated in the dark for $48 \mathrm{~h}$ at $+33^{\circ} \mathrm{C}$, relative humidity $85 \%$ prior to development of GFP fluorescence visible when illuminated with long wave, $395 \mathrm{~nm}$, ultraviolet light illumination (Fig. 2a) and were offered for cannibalization (Experiments A and B).

Analysis of virus replication. Total RNA was extracted from adult honey bee workers or pupae, which were flash-frozen and stored at $-80^{\circ} \mathrm{C}$. RNA extraction from individual insects included homogenization with $1 \mathrm{~mL}$ of Trizol reagent (Ambion) and further purification using RNeasy kits (QIAGEN) according to the manufacturer's instructions. Extraction of total RNA from pools of frozen worker bees started with lysis in guanidine isothiocyanate buffer as described previously ${ }^{61}$, followed by further disruption using QIAShredder (QIAGEN) and purification using RNeasy kits (QIAGEN). Quantification of DWV and GFP RNA in these RNA extracts was carried out by RT-qPCR as previously ${ }^{44}$ and included cDNA synthesis using Superscript III (Invitrogen) and random hexanucleotides as primers, and qPCR using SYBR green (BioRad) and the primers specific to DWV genomic RNA (5'-GAGATCGAAGCGCATGAACA-3' and 5'-TGAATTCAGTGTCGCCCATA-3', positions 6497-6626 nt of DWV, positions 7268-7397 of DWV-L-GFP), to the region spanning the eGFP-structural VP2 interface (GFP-specific primer 5'-GCATGGACGAGCTGTACAAG-3', and DWV-specific 5'-CCTTTTCTA ATTCAACTTCACC-3', positions 2526-2624 of DWV-L-GFP genome), and to the honey bee $\beta$-actin mRNA (5'-AGGAATGGAAGCTTGCGGTA-3' and 5'-AATTTTCATGGTGGATGGTGC-3'). The plasmid pDWV-L$\mathrm{GFP}^{44}$ was used as a standard for quantification of DWV and GFP copy numbers, which were log-transformed 
prior to statistical analyses. One-way analysis of variance (ANOVA) and Tukey's HSD post-hoc tests were used to assess the significance of the differences among the treatment groups.

The cDNA was used to amplify a 1237 nt cDNA fragments corresponding to the 5 ' region of DWV RNA (30-1266 nt) containing the PacI site introduced into the clone-derived DWV-L-GFP, but absent in the wildtype DWV, using primers 5'-GCCTTCCATAGCGAATTACG-3' and 5'-CGCCGCCTGGCTTCATCA-3'. The amplicons were digested with PacI restriction enzyme (NEB) for $2 \mathrm{~h}$, separated by agarose gel electrophoresis and the images were used to estimate the proportion of clone-derived DWV using Image ${ }^{62}$.

Received: 27 November 2020; Accepted: 12 April 2021

Published online: 26 April 2021

\section{References}

1. Grassly, N. C. \& Fraser, C. Mathematical models of infectious disease transmission. Nat. Rev. Microbiol. 6, 477-487 (2008).

2. Cressler, C. E., McLeod, D. V., Rozins, C., Van Den Hoogen, J. \& Day, T. The adaptive evolution of virulence: A review of theoretical predictions and empirical tests. Parasitology 143, 915-930 (2016).

3. Lanzi, G. et al. Molecular and biological characterization of deformed wing virus of honeybees (Apismellifera L.). J. Virol. 80, 4998-5009 (2006).

4. Dainat, B., Evans, J. D., Chen, Y. P., Gauthier, L. \& Neumann, P. Dead or alive: Deformed wing virus and Varroa destructor reduce the life span of winter honeybees. Appl. Environ. Microbiol. 78, 981-987 (2012).

5. Highfield, A. C. et al. Deformed wing virus implicated in overwintering honeybee colony losses. Appl. Environ. Microbiol. 75, 7212-7220 (2009).

6. Le Conte, Y., Ellis, M. \& Ritter, W. Varroa mites and honey bee health: Can Varroa explain part of the colony losses?. Apidologie 41, 353-363 (2010).

7. De Miranda, J. R. \& Genersch, E. Deformed wing virus. J. Invertebr. Pathol. 103, S48-S61 (2010).

8. Martin, S. J. \& Brettell, L. E. Deformed wing virus in honeybees and other insects. Annu. Rev. Virol. 6, 49-69 (2019).

9. Sumpter, D. J. \& Martin, S. J. The dynamics of virus epidemics in Varroa-infested honey bee colonies. J. Anim. Ecol. 73, 51-63 (2004).

10. Ramsey, S. D. et al. Varroa destructor feeds primarily on honey bee fat body tissue and not hemolymph. Proc. Natl. Acad. Sci. 116, 1792-1801 (2019).

11. Yang, X. \& Cox-Foster, D. L. Impact of an ectoparasite on the immunity and pathology of an invertebrate: Evidence for host immunosuppression and viral amplification. Proc. Natl. Acad. Sci. 102, 7470-7475 (2005).

12. Rosenkranz, P., Aumeier, P. \& Ziegelmann, B. Biology and control of Varroa destructor. J. Invertebr. Pathol. 103, S96-S119 (2010).

13. Wilfert, L. et al. Deformed wing virus is a recent global epidemic in honeybees driven by Varroa mites. Science 351, 594-597 (2016).

14. Dalmon, A. et al. Evidence for positive selection and recombination hotspots in deformed wing virus (DWV). Sci. Rep. 7, 1-12 (2017).

15. Martin, S. J. et al. Global honey bee viral landscape altered by a parasitic mite. Science 336, 1304-1306 (2012).

16. Moore, J. et al. Recombinants between deformed wing virus and Varroa destructor virus-1 may prevail in Varroa destructor-infested honeybee colonies. J. Gen. Virol. 92, 156-161 (2011).

17. Ryabov, E. V. et al. A virulent strain of deformed wing virus (DWV) of honeybees (Apis mellifera) prevails after Varroa destructormediated, or in vitro, transmission. PLoS Pathog. 10, e1004230 (2014).

18. Ryabov, E. V. et al. Dynamic evolution in the key honey bee pathogen deformed wing virus: Novel insights into virulence and competition using reverse genetics. PLoS Biol. 17, e3000502 (2019).

19. Mondet, F. et al. Specific cues associated with honey bee social defence against Varroa destructor infested brood. Sci. Rep. 6, 25444 (2016).

20. Spivak, M. \& Danka, R. G. Perspectives on hygienic behavior in Apismellifera and other social insects. Apidologie https://doi.org/ $10.1007 /$ s13592-020-00784-z (2020).

21. Spivak, M. \& Gilliam, M. Facultative expression of hygienic behaviour of honey bees in relation to disease resistance. J. Apic. Res. 32, 147-157 (1993).

22. Baracchi, D., Fadda, A. \& Turillazzi, S. Evidence for antiseptic behaviour towards sick adult bees in honey bee colonies. J. Insect Physiol. 58, 1589-1596 (2012).

23. Traynor, K. S. et al. Varroa destructor: A complex parasite, crippling honey bees worldwide. Trends Parasitol. 36, 592-606 (2020).

24. Sun, Q. \& Zhou, X. Corpse management in social insects. Int. J.: Biol. Sci. 9, 313 (2013).

25. Van Allen, B. G. et al. Cannibalism and infectious disease: Friends or foes?. Am. Nat. 190, 299-312 (2017).

26. Bourke, A. F. Queen behaviour, reproduction and egg cannibalism in multiple-queen colonies of the ant Leptothorax acervorum. Anim. Behav. 42, 295-310 (1991).

27. Pulliainen, U., Helanterä, H., Sundström, L. \& Schultner, E. The possible role of ant larvae in the defence against social parasites. Proc. R. Soc. B 286, 20182867 (2019).

28. Evans, H. \& West-Eberhard, M. The Wasps (Univ. Michigan, 1970).

29. Schmickl, T. \& Crailsheim, K. Cannibalism and early capping: Strategy of honeybee colonies in times of experimental pollen shortages. J. Comp. Physiol. A 187, 541-547 (2001).

30. Webster, T. C., Peng, Y. S. \& Duffey, S. S. Conservation of nutrients in larval tissue by cannibalizing honey bees. Physiol. Entomol. 12, 225-231 (1987).

31. Woyke, J. Cannibalism and brood-rearing efficiency in the honeybee. J. Apic. Res. 16, 84-94 (1977).

32. Chouvenc, T. Limited survival strategy in starving subterranean termite colonies. Insectes Soc. 67, 71-82 (2020).

33. Raina, A. K., Park, Y. I. \& Lax, A. Defaunation leads to cannibalism in primary reproductives of the Formosan subterranean termite, Coptotermes formosanus (Isoptera: Rhinotermitidae). Ann. Entomol. Soc. Am. 97, 753-756 (2004).

34. Schmickl, T. \& Crailsheim, K. Inner nest homeostasis in a changing environment with special emphasis on honey bee brood nursing and pollen supply. Apidologie 35, 249-263 (2004).

35. Meunier, J. Social immunity and the evolution of group living in insects. Philos. Trans. R. Soc. B Biol. Sci. 370, 20140102 (2015).

36. Rueppell, O., Hayworth, M. K. \& Ross, N. Altruistic self-removal of health-compromised honey bee workers from their hive. J. Evol. Biol. 23, 1538-1546 (2010).

37. Halling, L. \& Oldroyd, B. P. Do policing honeybee (Apis mellifera) workers target eggs in drone comb?. Insectes Soc. 50, 59-61 (2003).

38. Santomauro, G., Oldham, N. J., Boland, W. \& Engels, W. Cannibalism of diploid drone larvae in the honey bee (Apis mellifera) is released by odd pattern of cuticular substances. J. Apic. Res. 43, 69-74 (2004).

39. Imdorf, A., Rickli, M., Kilchenmann, V., Bogdanov, S. \& Wille, H. Nitrogen and mineral constituents of honey bee worker brood during pollen shortage. Apidologie 29, 315-325 (1998). 
40. Rudolf, V. H. \& Antonovics, J. Disease transmission by cannibalism: Rare event or common occurrence?. Proc. R. Soc. B Biol. Sci. 274, 1205-1210 (2007).

41. Chapman, J. W. et al. Age-related cannibalism and horizontal transmission of a nuclear polyhedrosis virus in larval Spodoptera frugiperda. Ecol. Entomol. 24, 268-275 (1999).

42. Hamano, K. et al. Waterborne and cannibalism-mediated transmission of the Yellow head virus in Penaeus monodon. Aquaculture 437, 161-166 (2015)

43. Möckel, N., Gisder, S. \& Genersch, E. Horizontal transmission of deformed wing virus: Pathological consequences in adult bees (Apis mellifera) depend on the transmission route. J. Gen. Virol. 92, 370-377 (2011).

44. Ryabov, E. V. et al. Development of a honey bee RNA virus vector based on the genome of a deformed wing virus. Viruses 12 , 374 (2020).

45. Posada-Florez, F. et al. Deformed wing virus type A, a major honey bee pathogen, is vectored by the mite Varroa destructor in a non-propagative manner. Sci. Rep. 9, 1-10 (2019).

46. Bull, J. C. et al. A strong immune response in young adult honeybees masks their increased susceptibility to infection compared to older bees. PLoS Pathog. 8, e1003083 (2012).

47. Shi, M. et al. Redefining the invertebrate RNA virosphere. Nature 540, 539-543 (2016).

48. Masterman, R., Ross, R., Mesce, K. \& Spivak, M. Olfactory and behavioral response thresholds to odors of diseased brood differ between hygienic and non-hygienic honey bees (Apis mellifera L.). J. Comp. Physiol. A 187, 441-452 (2001).

49. Crailsheim, K. Trophallactic interactions in the adult honeybee (Apis mellifera L.). Apidologie 29, 97-112 (1998).

50. Nixon, H. \& Ribbands, C. R. Food transmission within the honeybee community. Proc. R. Soc. Lond. Ser. B Biol. Sci. 140, 43-50 (1952).

51. Arathi, H. \& Spivak, M. Influence of colony genotypic composition on the performance of hygienic behaviour in the honeybee, Apis mellifera L. Anim. Behav. 62, 57-66 (2001).

52. Knecht, D. \& Kaatz, H. Patterns of larval food production by hypopharyngeal glands in adult worker honey bees. Apidologie 21, 457-468 (1990).

53. Li, Z. et al. Transcriptional and physiological responses of hypopharyngeal glands in honeybees (Apis mellifera L.) infected by Nosema ceranae. Apidologie 50, 51-62 (2019).

54. Lass, A. \& Crailsheim, K. Influence of age and caging upon protein metabolism, hypopharyngeal glands and trophallactic behavior in the honey bee (Apis mellifera L.). Insectes Soc. 43, 347-358 (1996).

55. Chiou, S.-S. \& Chen, W.-J. Mutations in the NS3 gene and 3'-NCR of Japanese encephalitis virus isolated from an unconventional ecosystem and implications for natural attenuation of the virus. Virology 289, 129-136 (2001).

56. Steel, A., Gubler, D. J. \& Bennett, S. N. Natural attenuation of dengue virus type-2 after a series of island outbreaks: A retrospective phylogenetic study of events in the South Pacific three decades ago. Virology 405, 505-512 (2010).

57. de Souza, F. S., Allsopp, M. H. \& Martin, S. J. Deformed wing virus prevalence and load in honeybees in South Africa. Arch. Virol. 166, 237-241 (2020).

58. Martin, S. J. et al. Varroa destructor reproduction and cell re-capping in mite-resistant Apis mellifera populations. Apidologie 51, 369-381 (2020).

59. Kulhanek, K. et al. Survey-derived best management practices for backyard beekeepers improve colony health and reduce mortality. PLOS ONE 16, e0245490 (2021).

60. Peck, D. T. \& Seeley, T. D. Mite bombs or robber lures? The roles of drifting and robbing in Varroa destructor transmission from collapsing honey bee colonies to their neighbors. PLOS ONE 14, e0218392 (2019).

61. Ryabov, E. V. et al. Recent spread of Varroa destructor virus-1, a honey bee pathogen, in the United States. Sci. Rep. 7, 1-10 (2017).

62. Abràmoff, M. D., Magalhães, P. J. \& Ram, S. J. Image processing with ImageJ. Biophoton. Int. 11, 36-42 (2004).

\section{Acknowledgements}

This research used resources provided by the SCINet project of the USDA-Agricultural Research Service, ARS project number 0500-00093-001-00-D and was supported by the USDA National Institute of Food and Agriculture Grant 2017-06481 to EVR, JDE, and YC. USDA is an equal opportunity provider and employer.

\section{Author contributions}

F.P.-F., J.D.E. and E.V.R., the corresponding authors, conceived the study and contributed equally to this research. Z.L. and D.H. carried out field monitoring of cannibalism. Y.C. supervised honeybee colony monitoring. F.P.-F., Z.L. and E.V.R. carried work with bees and mites. E.V.R. and F.P.-F. carried out the laboratory molecular work and analyzed virus quantification. M.H. analyzed accumulation of the honey bee transcripts. F.P.-F., Z.L., J.D.E. and E.V.R., wrote the manuscript. All co-authors contributed to data interpretation, and to the writing of the manuscript.

\section{Competing interests}

The authors declare no competing interests.

\section{Additional information}

Supplementary Information The online version contains supplementary material available at https://doi.org/ 10.1038/s41598-021-88649-y.

Correspondence and requests for materials should be addressed to F.P.-F., J.D.E. or E.V.R.

Reprints and permissions information is available at www.nature.com/reprints.

Publisher's note Springer Nature remains neutral with regard to jurisdictional claims in published maps and institutional affiliations. 
(c) (i) Open Access This article is licensed under a Creative Commons Attribution 4.0 International (c) License, which permits use, sharing, adaptation, distribution and reproduction in any medium or format, as long as you give appropriate credit to the original author(s) and the source, provide a link to the Creative Commons licence, and indicate if changes were made. The images or other third party material in this article are included in the article's Creative Commons licence, unless indicated otherwise in a credit line to the material. If material is not included in the article's Creative Commons licence and your intended use is not permitted by statutory regulation or exceeds the permitted use, you will need to obtain permission directly from the copyright holder. To view a copy of this licence, visit http://creativecommons.org/licenses/by/4.0/.

This is a U.S. Government work and not under copyright protection in the US; foreign copyright protection may apply 2021 